\title{
CONCORDÂNCIA ENTRE O ÍNDICE DE MASSA CORPORAL DE QUÉTELET E O DE TREFETHEN NA ESTIMATIVA DO ESTADO NUTRICIONAL DE ESCOLARES
}

Cleberson Ferreira*, Antonio Roberto Robles**, Luis Paulo Gomes Mascarenhas***, Valderi Abreu de Lima $^{* * * *}$, Wallace Bruno de Souza ${ }^{* * * * *}$, William Cordeiro de Souza ${ }^{* * * * * *}$

\footnotetext{
Autor correspondente: William Cordeiro de Souza - williammixx@hotmail.com

* Graduando em Educação Física pela Universidade do Contestado - UnC

** Departamento de Educação Física da Universidade do Contestado - UnC

*** Doutor em Saúde da Criança e do Adolescente. Professor da Universidade Estadual do Centro-Oeste

**** Mestrando em Educação Física pela Universidade Federal do Paraná

***** Universidade do Vale do Itajaí - Univali

****** Professor de Educação Física Escolar na Prefeitura do Município de Três Barras - SC
}

\section{Resumo}

Introdução: Constantemente diversos métodos antropométricos vêm sendo desenvolvidos com o intuito de avaliar o estado nutricional tanto de adultos, quanto de crianças e adolescentes. Objetivo: O presente estudo objetivou-se em verificar o nível de concordância entre o índice de massa corporal (IMC) de Quételet e o de Trefethen na estimativa do estado nutricional de escolares. Métodos: A amostra intencional foi constituída por 113 escolares (48 meninas e 65 meninos) com idades entre 10 e 15 anos, do município de Canoinhas/SC. Para avaliação antropométrica foram coletados os dados de massa corporal e estatura. Através desses foi calculado o IMC, proposto por Quételet (1870) e o IMC sugerido por Trefethen (2013). Para verificar a normalidade dos dados foi utilizado o teste de Kolmogorov-Smirnov. Após, realizou-se a estatística descritiva. O fator de correlação de Pearson ( $r$ ) foi realizado para verificar as associações entre os métodos. Recorreu-se ao teste Qui-quadrado para verificar as diferenças entre as frequências percentuais. Para analisar o nível concordância entre os métodos foi realizado o método de Bland-Altman (1986). Resultados: Foi observada uma relação muito forte e significante entre as variáveis analisadas $(r=0,98 ; p=<0,001)$ com um intervalo de confiança de (IC 95\% = 0,97-0,98) e através do método Bland-Altman (1986) pode-se perceber que as variáveis analisadas apresentaram concordâncias entre si. Conclusão: Ao finalizar o estudo, foi verificado que o IMC de Quételet e o de Trefethen apresentam concordância na estimativa do estado nutricional de escolares.

Palavras-chave: Concordância; Estado Nutricional; Escolares. 


\title{
COMPARISION BETWEEN QUÉTELET AND TREFETHEN BODY MASS INDEXES IN ESTIMATING NUTRITION OF CHILDREN IN SCHOOL AGE
}

\begin{abstract}
Introduction: Constantly various anthropometric methods have been developed in order to evaluate the nutritional status of adults, children and adolescents. Objective: This study aimed to assess similarities between the body mass index (BMI) of Quételet and Trefethen to estimate the nutritional status of children in school age. Methods: The sample consisted of 113 students ( 48 girls and 65 boys) aged between 10 and 15 years, in the city of Canoinhas/SC. Anthropometrics was collected by measuring body mass and height. With those, two types of BMI were calculated, as proposed by Quételet (1870) and as suggested by Trefethen (2013). To check the normality of the data the Kolmogorov-Smirnov test was employed. Afterwards, the descriptive statistics were calculated. The Pearson correlation factor $(r)$ was performed to verify similarities between the methods. The chi-square test was employed to find possible differences among the percentage frequencies. To analyze the level of similarity between the methods the Bland-Altman method was applied (1986). Results: We observed a strong and significant relation between the variables $(r=0.98, p=<0.001)$ with a confidence interval of ( $95 \% \mathrm{Cl}=0.97$ to 0.98 ) and using the Bland-Altman method (1986) can be seen that the variables analyzed showed consistencies between them. Conclusion: At the end of the study, it was found that the Quételet BMI and the Trefethen present similarities in estimating the nutritional status of schoolchildren.

Kepwords: Agreement; Nutritional status; School.
\end{abstract}

\section{INTRODUÇÃO}

O Índice de Massa Corporal (IMC), também conhecido como índice de Quételet, em respeito ao seu criador Adolphe Quételet, é um método antropométrico muito utilizado para avaliar o estado nutricional de grandes populações, o mesmo é obtido através da divisão da massa corporal em quilogramas, pela estatura em metro, elevada ao quadrado $\left(\mathrm{kg} / \mathrm{m}^{2}\right)$. Em suas pesquisas, Quételet observou que após o término do crescimento, ou seja, na vida adulta, o peso de indivíduos de tamanho normal era proporcional ao quadrado da estatura. ${ }^{(1,2)}$

Apesar do IMC ser bastante utilizado em estudos epidemiológicos, apresentar baixo custo e simplicidade para realização das medidas, o mesmo apresenta controversas quanto a sua reprodutibi- lidade, já que ele não é capaz de fornecer informações sobre a composição corporal e a distribuição da gordura corporal. ${ }^{(3,4)}$

Para Vieira(5) e Giuntoli(6) o IMC é um índice para se aferir excesso de peso e não de gordura corporal, por isso continua a discussão em torno da sua capacidade preditiva na identificação gordura corporal elevada. Mas cabe mencionar que o IMC apresenta boa associação com o risco de mortalidade e morbidade das doenças crônicas. (3,7,8)

Um dos grandes críticos da equação proposta por Quételet é o professor inglês Floyd Nicholas Trefethen, que propõem uma nova equação para estimar o IMC, segundo o matemático essa nova formula apresenta uma melhor aproximação do ta- 
manho e das formas corporais, sendo que a mesma representa as dimensões corporais de maneira errônea, uma vez que pessoas de maior estatura possuem compartimentos estruturais e fisiológicos maiores que pessoas de menor estatura. $(9,10)$

Sendo assim, o presente estudo objetivou-se em verificar o nível de concordância entre o IMC de Quételet e o de Trefethen na estimativa do estado nutricional de escolares.

\section{MÉTODOS}

A presente pesquisa seguiu as Diretrizes e Normas Regulamentadoras de Pesquisa envolvendo Seres Humanos, Resolução - CNS 466/12, submetido a análise do comitê de ética da Universidade do Contestado (UnC) e da Plataforma Brasil sob o número CAAE: 05020512.7.0000.0117. Cabe mencionar que os escolares somente foram avaliados mediante assinatura do Termo de Consentimento Livre e Esclarecido pelos pais e responsáveis.

Sendo assim, a amostra intencional do presente estudo foi constituída por 113 escolares (48 meninas e 65 meninos) com idades entre 10 e 15 anos, matriculadas regularmente no ensino fundamental de uma escola do município de Canoinhas - SC.

Foram excluídos do estudo adolescentes portadores de doenças crônicas que interferissem diretamente com a massa corporal e a estatura e aqueles que, no momento da avaliação, tivessem algum impedimento para a obtenção das medidas antropométricas, que não compareceram na escola nos dias marcados para coleta de dados, e os adolescentes cujos pais não autorizaram a participação. Sendo também respeitada a vontade dos adolescentes que se recusaram a participar mesmo com a autorização dos pais.

A avaliação antropométrica consistiu na mensuração da massa corporal e estatura. $\mathrm{Na}$ avaliação da massa corporal os escolares permaneciam descalços, vestindo roupas leves, posicionados em pé, com o peso igualmente distribuído em ambos os pés. ${ }^{(11)} \mathrm{Na}$ avaliação da massa corporal foi utilizada uma balança digital da marca Techline, (fabricada no Brasil) devidamente calibrada, com graduação de 100 gramas e escalas variando de o a $180 \mathrm{Kg}$.

A estatura foi mensurada com os escolares descalços, em posição anatômica, sob a base da fita métrica, encostada à parte posterior do corpo e a cabeça posicionada no plano de "Frankfurt", estando em apneia inspiratória no momento da medida. (11) A estatura foi obtida através de uma trena flexível marca Sanny Medical Sparrett, (fabricada no Brasil), fixada na parede lisa, com 3 metros e graduação de $0,1 \mathrm{~cm}$ com o zero coincidindo com o solo.

Através dos dados de massa corporal e estatura foi calculado o IMC, proposto por Quételet ${ }^{(12)}$ e o IMC sugerido por Trefethen ${ }^{(10)}$ utilizado às seguintes formulas: IMC Quételet= Massa Corporal/Estatura $^{2}$ e IMC Trefethen= Massa corporal (Kg) x 1,3/ altura $(\mathrm{m})^{2,5}\left(\mathrm{kgxi}, 3 / \mathrm{m}^{2,5}\right)$.

Para a classificação do IMC utilizou-se como referência as curvas de percentis, recomendado por Conte e Monteiro ${ }^{(13)}$ que indicaram como ponto de corte valores $<17,5 \mathrm{~kg} / \mathrm{m}^{2}$ para baixo peso, $\geq 25 \mathrm{~kg} /$ $\mathrm{m}^{2}$ para sobrepeso e $\geq 3 \mathrm{okg} / \mathrm{m}^{2}$ para obesidade.

Para verificar a normalidade dos dados foi utilizado o teste de Kolmogorov-Smirnov. Visto que a distribuição foi normal, realizou-se a estatística descritiva (média, desvio padrão e a (\%) frequência percentual). O fator de correlação de Pearson ( $r$ ) foi realizado para verificar as associações entre o IMC Quételet e o IMC Trefethen. Recorreu-se ao teste Qui-quadrado para verificar as diferenças entre as frequências percentuais das classificações obtidas entre os métodos. Para analisar o nível concordância entre o IMC Quételet e o IMC Trefethen foi realizado o método de Bland-Altman. ${ }^{(14)}$ Foi adotado um nível de significância de $p<0,05$. As análises foram realizadas no software MedCalc Statistical.

\section{RESULTADOS}

A tabela 1 apresenta os dados de idade, massa corporal, estatura, IMC (Quételet) e de IMC (Trefethen) para caracterização da amostra. 
Tabela 1 - Caracterização dos escolares do município de Canoinhas/SC, 2016

\begin{tabular}{lcc}
\hline \multicolumn{1}{c}{ VARIÁVEIS } & MÉDIA & DESVIO PADRÃO \\
\hline Idade (anos) & 12,6 & 1,4 \\
Massa Corporal $(\mathrm{kg})$ & 49,86 & 13,40 \\
Estatura $(\mathrm{m})$ & 1,58 & 0,11 \\
IMC Quételet $\left(\mathrm{kg} / \mathrm{m}^{2}\right)$ & 19,51 & 3,88 \\
IMC Trefethen $\left(\mathrm{Kgxl}, 3 / \mathrm{m}^{2,5}\right)$ & 20,12 & 3,86 \\
\hline
\end{tabular}

O gráfico 1 apresenta a relação entre o IMC de Quételet e o IMC de Trefethen. E se pode perceber uma relação muito forte e significante entre as variáveis analisadas $(\mathrm{r}=0,98 ; \mathrm{p}=<0,001)$ com um intervalo de confiança de (IC $95 \%=0,97-0,98)$.

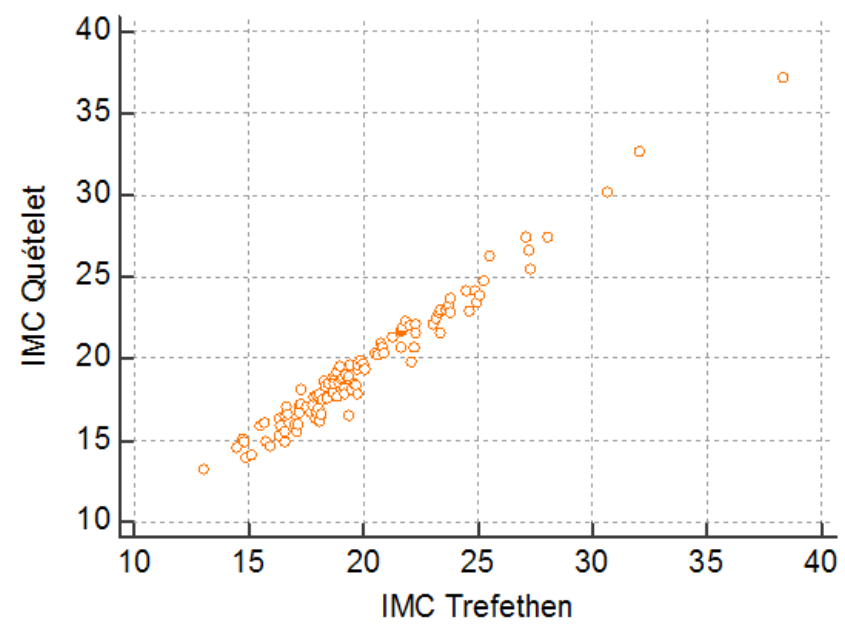

Gráfico 1 - Relação entre o IMC de Quételet e o IMC de Trefethen dos escolares do município de Canoinhas/SC, 2016

O gráfico 2 apresenta o nível de concordância entre o IMC de Quételet e o IMC de Trefethen. E através do método Bland-Altman ${ }^{(14)}$ pode-se perce- ber que as variáveis analisadas apresentaram concordâncias entre si.

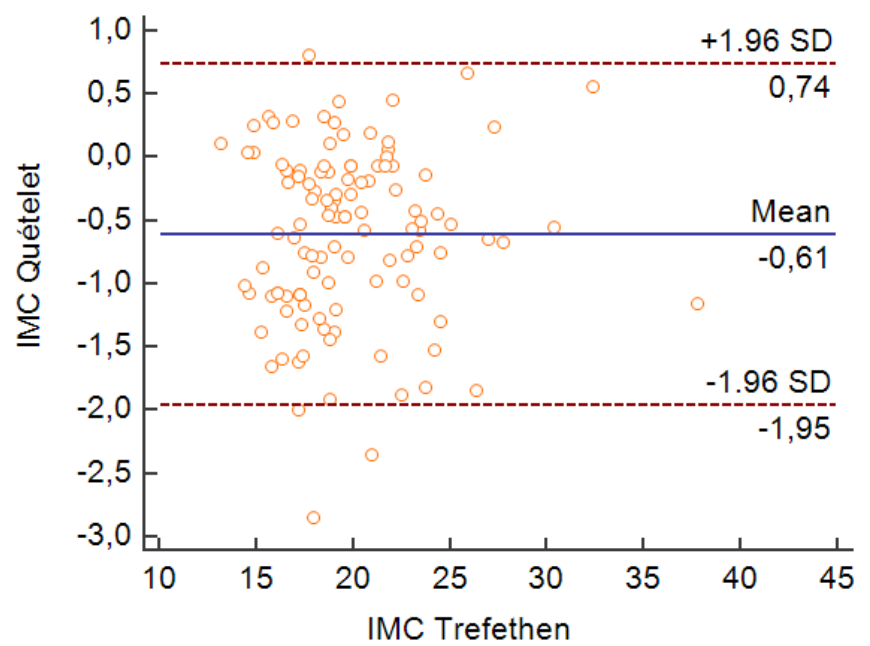

Gráfico 2 - Concordância entre o IMC de Quételet e o IMC de Trefethen dos escolares do município de Canoinhas/ SC, 2016 
O gráfico 3 apresenta as comparações percentuais das classificações obtidas nos métodos do IMC. E pode-se verificar que não foram encontra- das diferenças significativas entre os métodos através do teste de Qui-quadrado $(p=0,481)$.

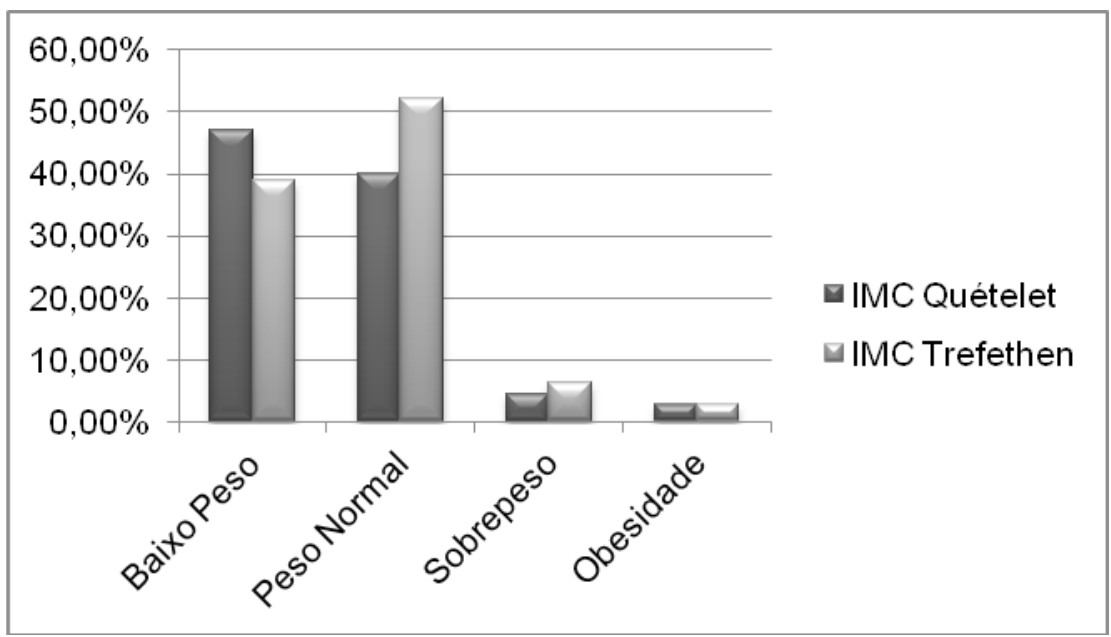

Gráfico 3 - Classificações obtidas no IMC dos escolares do município de Canoinhas/SC, 2016

\section{DISCUSSÃO}

O presente estudo com o objetivo de verificar o nível de concordância entre o IMC de Quételet e o de Trefethen na estimativa do estado nutricional de escolares verificou uma relação muito forte e significante entre IMC de Quételet e o de Trefethen. Diante dos resultados apresentados foi possível observar que as variáveis analisadas apresentaram concordâncias entre si através do método de Bland-Altman. ${ }^{(14)}$ Cabe mencionar que as comparações percentuais das classificações obtidas nos métodos do IMC não apresentaram diferenças significativas.

Em estudo semelhante realizado por Ribas $/ r^{(15)}$ com o objetivo de verificar o nível de aplicabilidade do IMC proposto por Trefethen, comparado a outras técnicas indiretas de avaliação da composição corporal de escolares foi encontrada uma relação muito forte e significante entre o IMC de Trefethen e o IMC de Quételet ( $r=0,97 ; p=<0,001)$. Já entre - IMC de Trefethen e o \%G foi verificada uma relação forte e significante $(r=0,72 ; p=<0,001)$. Diante dos dados encontrados os autores concluíram que a nova equação do IMC pode ser considerada como um novo método para avaliar o estado nutricional de crianças e adolescentes.

Em estudo similar desenvolvido por Westphal( ${ }^{(16)}$ com objetivo de relacionar o IMC de Quételet e - IMC proposto por Trefethen em acadêmicos de Educação Física foi encontrada uma relação muito forte e significante $(r=0,99 ; p=<0,001)$; com um intervalo de confiança de (IC 95\%=0,96-0,99) entre os métodos. Já nas comparações das porcentagens obtidas nas classificações de ambos os métodos não foram encontradas diferenças significativas entre os resultados apresentados. Fato observado no presente estudo. Sendo assim, o estudo supracitado concluiu que a nova equação do IMC apresenta-se como uma nova ferramenta na avaliação do estado nutricional.

Vale ressaltar que a validação da equação desenvolvida por Trefethen foi realizada através da análise de regressão entre a equação de Quételet e foi encontrada uma associação positiva e perfeita $\left(R^{2}=1,0\right) \cdot{ }^{(10)}$ Segundo Fernandes ${ }^{(17)}$ os resultados da aplicação desta nova formula pode garantir aos indivíduos mais baixos 1 ponto mais de IMC e aos mais altos 1 ponto menos, quando comparado ao IMC de Quételet. 
Sendo assim, a equação proposta por Trefethen mostra-se como uma nova ferramenta para avaliar o estado nutricional tanto de adultos, quanto de crianças, onde essa formula apresenta dados mais próximos da realidade. ${ }^{(16,17)}$

O presente estudo limitou-se em não controlar o nível de atividade física da população estudada, bem como a classe socioeconômica, possivelmente estes fatores podem ter influenciado nos achados do estudo. Assim, destaca-se a necessidade da realização de novos estudos, com distintas populações utilizando a nova equação para assim dar mais fidedignidade ao novo método de avaliação de estado nutricional.

\section{CONCLUSÃO}

Ao finalizar o presente estudo, constatou-se uma relação muito forte e significante entre as propostas do IMC e através do método Bland-Altman pode-se perceber que as variáveis analisadas apresentaram concordâncias entre si. Desta forma, pode-se concluir que o IMC Trefethen pode ser um método aplicável para estimar o estado nutricional de escolares.

\section{REFERÊNCIAS}

1. Cervi A, Franceschini SCC, Priore SE. Análise crítica do uso do índice de massa corporal para idosos. Rev Nutr. 2005;18(6):765-75.

2. Pich S. Adolphe Quetelet e a biopolítica como teologia secularizada. Hist Ciênc Saúde Manguinhos. 2013;2O(3):849-64.

3. Rezende F, Rosado L, Franceschinni S, Rosado G, Ribeiro R, Marins JCB. Revisão crítica dos métodos disponíveis para avaliar a composição corporal em grandes estudos populacionais e clínicos. Arch Latinoam Nutr. 2007;57(4):327. 34.

4. Pedrosa CR. Métodos de Composição Corporal. Hermes Pardini Medicina Diagnóstica e Preventiva. 2014;2(8):1-2.
5. Vieira ACR, Alvarez MM, Marins VMR, Sichieri $R$, Veiga GV. Desempenho de pontos de corte do índice de massa corporal de diferentes referências na predição de gordura corporal em adolescentes. Cad Saúde Pública. 2006;22(8):1681-90.

6. Giuntoli ABB, Outa K, Monteiro PMA, Monomi PY, Morares SZ, Stulbach T. Comparação entre o índice de massa corpórea e porcentagem de gordura, e análise da relação cintura/quadril em desportistas de uma academia de São Paulo. Revista Digital. 2012;17(171):1.

7. Anjos L. A. Índice de massa corporal (massa corporal.estatura-2) como indicador do estado nutricional de adultos: revisão da literatura. Rev Saúde Pública. 1992;26(6):431-6.

8. Gonzalez AB, Hartge P, Cerhan JR, Flint AJ, Hannan L, Maclnnis RJ. et al. Body-Mass Index and Mortality among 1.46 Million White Adults. N Engl J Med. 2010;363(23):2211-9.

9. Buss V, Franz LBB, Steinmetz SB, Manenti M, Weber ITS, Conte FA. Estado nutricional: Análise de uma nova proposta de equação. In: Resumo no Salão do Conhecimento. ljuí, Rio Grande do Sul; $2 \mathrm{Ol3}$.

10. Trefethen LN. (BMI) Body mass index [Internet]. Oxford: Mathematical Institute of University of Oxford; 2013 [cited 2016 Feb 19]. Available from: https://people.maths.ox.ac.uk/trefethen/ bmi.html

11. Tritschler K. Medida e avaliação em educação física e esportes de Barrow \& McGee. $5^{\mathrm{a}}$ ed. Barveri: Manole; 2003.

12. Quételet A. Antropométrie ou mesure des différentes facultés de l'homme. Bruxelles, C. Muquardt, 1870.

13. Conde WL, Monteiro CA. Valores críticos do índice de massa corporal para classificação do estado nutricional de crianças e adolescentes brasileiros. J Pediatr. 2006;86(4):266-72.

14. Bland JM, Altman DG. Statistical methods for assessing agreement between two methods of clinical measurement. Lancet. 1986;8(8476):307-10. 
15. Ribas Jr MA, Mascarenhas LPG, Cordova M, Lima VA, Grzelczak MT, Souza WC.

Aplicabilidade do IMC de Trefethen em escolares. Revista Pesquisa em Fisioterapia. 2016;6(2):91-8.

16. Westphal P, Ferreira C, Adamczeski M, Camargo L, Santos R, Massaneiro AC. et al. Relação entre índice de massa corporal de Quételet e o de Trefethen. Revista CPAQV. 2016;8(3):1-6.
17. Fernandes MAC. Efeitos do índice de massa corporal e aptidão aeróbia no rendimento escolar em alunos do $3^{\circ}$ ciclo e ensino secundário. Vila Real: Universidade de Trás-osMontes e Alto Douro; 2015. 